\title{
Intraosseous Lipoma with Subsequent Esophageal Carcinoma Metastasis*
}

\author{
Singh Ramandeep", Madewell John ${ }^{1 \#}$, Rao Priya ${ }^{2}$, Bean Gregg ${ }^{1}$, Czerniak Bogdan $^{2}$, Lin Patrick $^{3}$ \\ ${ }^{1}$ Department of Musculoskeletal Radiology, University of Texas MD Anderson Cancer Center, Houston, USA \\ ${ }^{2}$ Department of Pathology, University of Texas MD Anderson Cancer Center, Houston, USA \\ ${ }^{3}$ Department of Orthopedic Oncology, University of Texas MD Anderson Cancer Center, Houston, USA \\ Email: \#jmadewell@mdanderson.org
}

Received February 2, 2012; revised May 1, 2012; accepted May 11, 2012

\begin{abstract}
Background: This case report describes a well documented proximal femoral metadiaphysis intraosseous lipoma which later developed metastasis from a new esophageal cancer. Metastatic disease to benign conditions is a rare finding. To the best of our knowledge, this is the first reported case of metastatic disease to an intraosseous lipoma. Case Description: The metastatic deposit was initially detected by plain-film radiography, performed to evaluate new onset right hip pain, as possible new cortical breakthrough with irregularity in the site of previously known proximal right femur intraosseous lipoma. Concurrent follow-up PET/CT study showed a new hypermetabolic focus within the known intraosseous lipoma indicating a new metastasis that was confirmed with an MRI as a new enhancing mass within the preexisting intraosseous lipoma. Subsequently, an MRI-guided biopsy and eventually surgical excision was performed providing the histological samples for radiologic-pathologic correlation. Purpose and Clinical Relevance: Clinicians need to be aware that unusual, complex patterns within benign lesions may be a reflection of unexpected conditions, such as insufficiency injury, malignant transformation and secondary metastatic disease, as exemplified by our case report.
\end{abstract}

Keywords: Intraosseous Lipoma; Esophageal Cancer; MRI; PET/CT; Metastasis

\section{Introduction}

Intraosseous lipomas are rare benign tumors of the bone that are most commonly found within the calcaneus and the proximal femur [1,2]. These lesions are classified according to their site of origin with intramedullary lipomas (IMLs) being the most common, followed by juxtacortical lipomas, intracortical lipomas, and subperiosteal lipomas which are the rarest types [2]. Histologically, the intraosseous lipomas are composed of mature adipose tissue along with varying amounts of fibrous and vascular tissues. In addition, they can involute resulting in foci of fat necrosis, cystic degeneration and dystrophic calcifications. Such appearance can closely resemble a bone infarct mandating meticulous radiologic-clinical correlation for an appropriate diagnosis [3].

The following is a case report of a patient with known esophageal carcinoma who developed a metastatic focus within a pre-existing proximal femoral intraosseous li-

\footnotetext{
*Conflict of interest statement: Authors of this document have no financial relationship with or conflicts of interests pertaining to this case report. The authors have full control of all primary data and agree to allow CORR to review data if requested. Work performed at UT MD Anderson Cancer Center.

${ }^{\#}$ Corresponding author.
}

poma. The presence of this metastatic deposit was detected on PET/CT examination and confirmed with an MRI. Subsequently, a histological specimen was obtained using MRI-guided biopsy and eventual surgical excision. To the best of our knowledge, this is the first reported case of metastatic disease to a pre-existing intraosseous lipoma.

\section{Case Report}

The patient was a 66-year-old male treated for a prior frontal sinus papilloma and benign fatty tumor right femur was noted. He was referred back to our clinic with abdominal discomfort and dysphagia. During work up an esophageal adenocarcinoma was diagnosed and patient underwent treatment with chemo-radiation therapy and esophagectomy. He was found to have an expansile, septated, lucent lesion in his right proximal femur on routine metastatic work-up imaging (Figure 1). This was identical to the prior CT. The patient had been experiencing intermittent non-debilitating right hip pain for 4 years. He did not seek medical consultation as the pain did not interfere with his activities of daily living. The initial PET/CT scan study demonstrated an expansile intramedullary proximal right femoral metadiaphyseal lesion 
with internal septations, no internal matrix, calcification, or cortical break-through (Figure 1). The lesion measured fat density $(\mathrm{HU}=-57.76)$ on the $\mathrm{CT}$ portion of the PET study that confirmed it to be an Intraosseous lipoma (Figure 1(B)). In addition, there was no FDG avidity (Figure 1(C)).

Six months later, the patient presented with markedly worse right hip pain. Pelvic and right hip radiographs demonstrated increased lucency within the expansile intramedullary lesion of the proximal femur. There was also noted more cortical thinning and a suggestion of cortical breakthrough, indicating a possible fracture secondary to a malignant or aggressive process (Figure 2). A concurrent follow-up PET/CT scan demonstrated a new FDG-avid focus in the subtrochanteric region within the previously seen intraosseous lipoma (Figure 3). This corresponded to a new soft tissue mass with different non-lipomatous characteristics $(\mathrm{HU}=35.89)$ on $\mathrm{CT}$ attenuation correction images (Figure 3). A follow-up contrast enhanced MRI confirmed the presence of an enhancing soft tissue focus within a pre-existing intraosseous lipoma (Figures 4(A)-(D)). The differential diagnosis included insufficiency fracture, malignant degeneration of the intraosseous lipoma [4], and metastasis from patient's known esophageal cancer. An MRI guided biopsy was subsequently performed, and the histological samples yielded metastatic esophageal carcinoma within a pre-existing intraosseous lipoma.

The right proximal femoral resection gross specimen demonstrated a well demarcated tumor involving the subtrochanteric intramedullary portion of the femoral metadiaphysis (Figure 5). Microscopically the tumor showed an infiltrative pattern of growth with invasion of the marrow fat with nuclear pleomorphism and mitotic activity (Figure 6(A)). Higher power view showed infiltration of the trabecular bone by adenocarcinoma. The

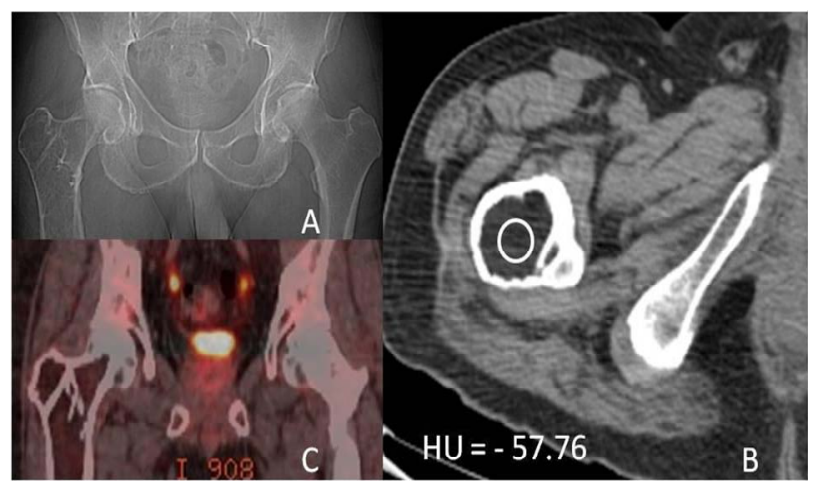

Figure 1. (A) AP scout radiograph of the Right Proximal Femur showing an expansile lytic lesion with no matrix calcifications; (B) Axial non-contrast CT demonstrating an expansile fatty lesion (HU $=-57.76)$ consistent with an Intraosseous lipoma; (C) Coronal PET/CT fusion images demonstrating no FDG avidity.

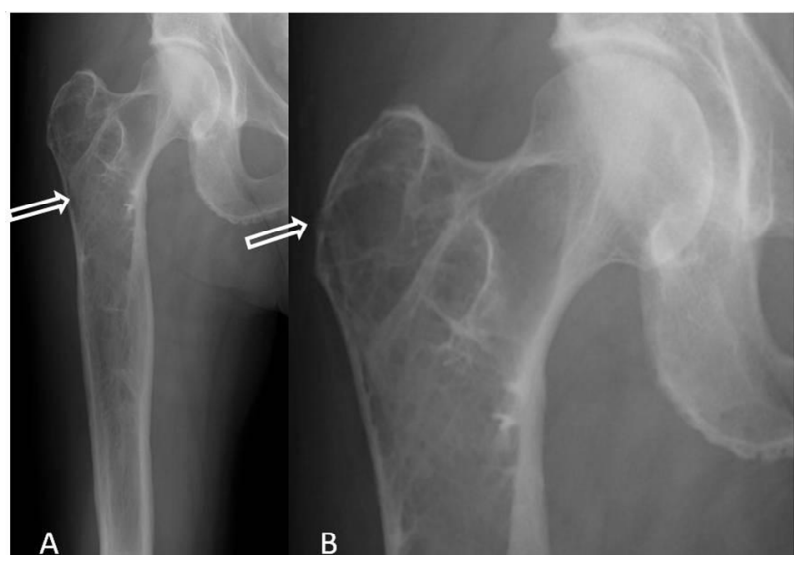

Figure 2. (A) AP radiograph of right hip showing known Intraosseous Lipoma, however, with a subtle new density in the subtrochanteric region (arrow); (B) Magnified coneddown AP view of right hip showing a focal cortical break in the greater trochanter (arrow).

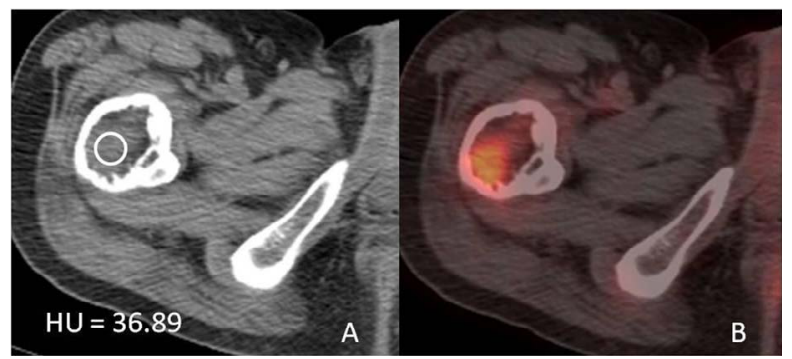

Figure 3. (A) Axial non-contrast CT demonstrating a soft tissue mass $(\mathrm{HU}=36.89)$ within the previously seen Intraosseous lipoma; (B) Axial PET/CT fusion images demonstrating new FDG avidity within this metastasis.

tumor cells are arranged in sheets and nests with a focal glandular patter, consistent with metastatic adenocarcinoma (Figure 6(B)).

\section{Discussion}

Intramedullary osseous lipoma (IML) is an uncommon primary bone tumor with a reported incidence of $0.1 \%$ $2.5 \%$ [5]. There is no sex predilection, and only about half of these patients are symptomatic and painful, while the others are discovered as incidental findings, as in our case on the routine PET/CT scan. The IMLs are more commonly found in the appendicular than the axial skeleton with the proximal femur being one of the most frequent sites for intraosseous lipomas along with the calcaneus $[1,3]$. In the long bones the IMLs are centered within the metaphysis and may occasionally extend into the diaphysis as is also seen in our case report.

Radiographically, IMLs present as lucent lesions with osseous expansion, trabecular thickening, and cortical thinning. They are frequently distinguished from the surrounding normal fatty marrow by a peripheral ossified 

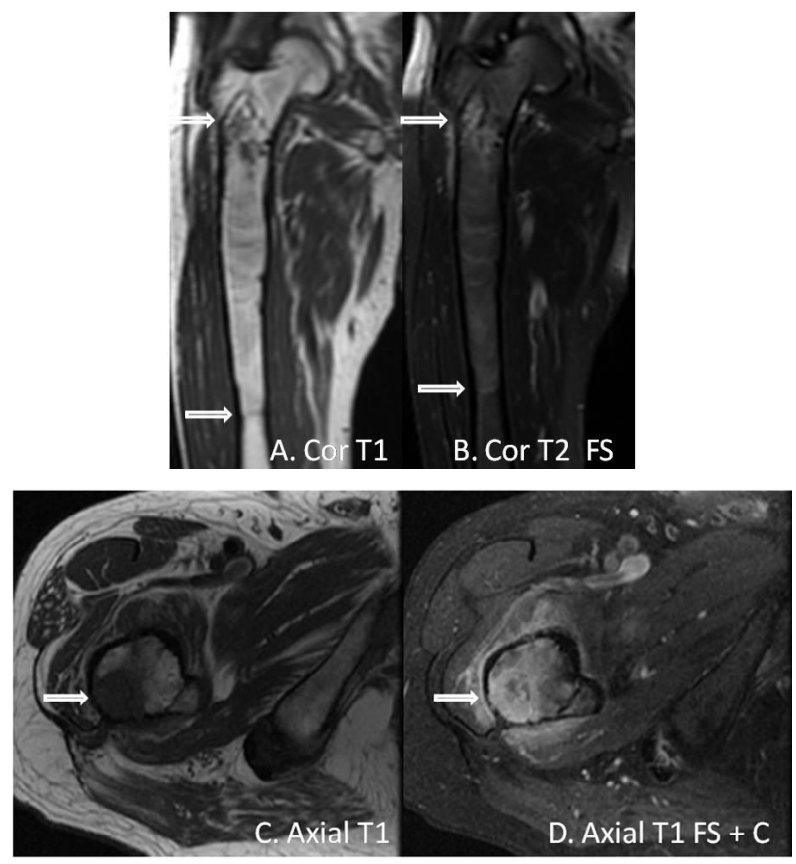

Figure 4. (A) Coronal T1 weighted-image demonstrating a proximal right femoral metadiaphysis lesion that is isointense to marrow with a thin cortical rim. A small hypointense lesion is present in the subtrochanteric region; (B) Coronal $\mathrm{T} 2$ fat saturation image demonstrating "fat dropout" indicating an intraosseous lipoma with the exception of a small focus in the subtrochanteric region; (C) and (D) Axial T1 weighted, and axial T1 weighted fat sat post contrast images demonstrating a sub-trochanteric focus with marked enhancement consistent with biopsy proven esophageal cancer metastasis to a pre-existing intraosseous lipoma.

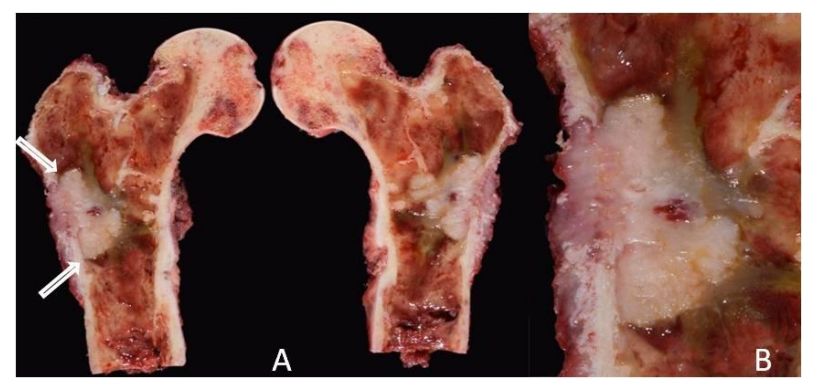

Figure 5. (A) Right proximal femoral resection showing a well demarcated tumor involving the subtrochanteric intramedullary portion of the femoral meta-diaphysis; (B) A close-up view of the tumor showing a grey-white rubbery solid cut surface.

rim or capsule. They may also contain cystic areas and dystrophic calcifications as the lipoma evolves. On CT scans, the attenuation value of normal fatty marrow is slightly greater than that of IMLs [6]. MRI is highly sensitive for detection of IMLs, which have signal intensity similar to that of subcutaneous adipose tissue on $\mathrm{T} 1$ and $\mathrm{T} 2$ weighted images and demonstrate fat "drop-out" on

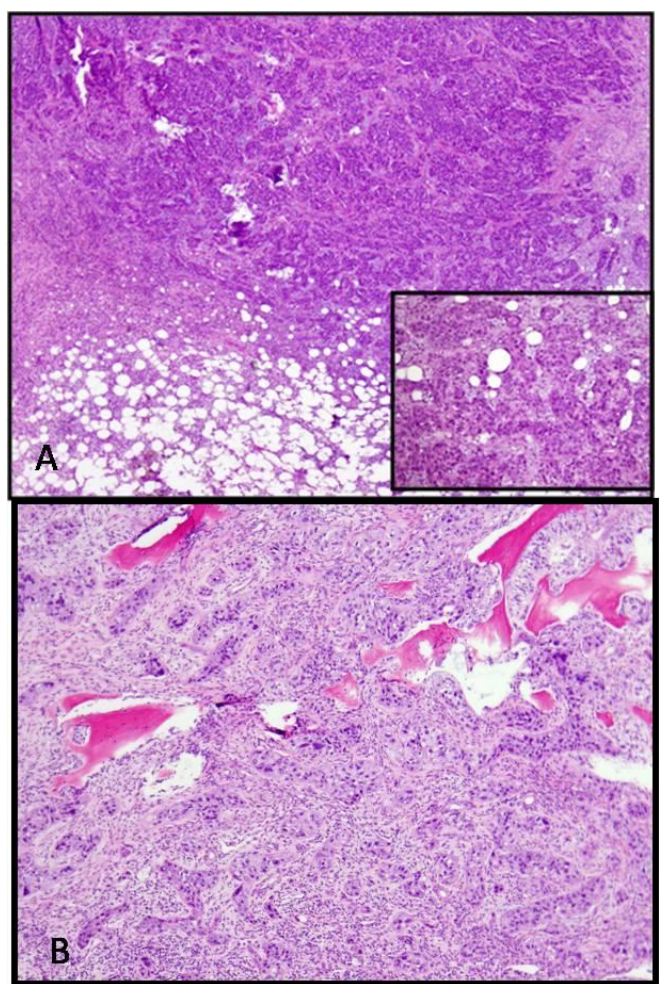

Figure 6. (A) Microscopically the tumor shows an infiltrative pattern of growth with invasion of the marrow fat. (Inset) Nuclear pleomorphism and mitotic activity were readily identified; (B) Higher power view showing infiltration of the trabecular bone by adenocarcinoma. The tumor cells are arranged in sheets and nests with a focal glandular patter, consistent with metastatic adenocarcinoma.

fat suppressed or STIR sequences [7-9]. In addition, the thin capsular rim can be clearly identified on the MRI. In our case, the multimodality imaging features at presentation were thought to be diagnostic of an IML, and therefore, a biopsy for histological confirmation was initially not felt to be necessary. The biopsy was subsequently performed since the follow-up imaging studies indicated a significant change in the lesion that suggested it was no longer a simple IML.

A few rare cases of malignant tissue within a pre-existing benign osseous tumor have been described. Exceedingly rare is the malignant transformation of the IMLs to malignant fibrous histiocytomas or liposarcomas as described by Milgram et al. [4]. Also, there is a case report describing an osseous collision-tumor involving breast carcinoma metastases to post-radiation scapular chondrosarcoma [10].

Esophageal carcinoma is the sixth leading cause of worldwide cancer related deaths. Esophageal carcinoma rarely metastasizes to bone with known incidence of 5\% $9 \%[11,12]$. Most of these osseous metastases occur in bones containing predominantly red marrow such as the spine, ribs, pelvis and the end of long bones especially 
proximal femora [13]. It may be more than pure coincidence that in our case, a tumor that uncommonly metastasizes to bone produced a metastatic lesion within an IML. The cellular microenvironment of the IML would be expected to be different from cancellous bone and might be more favorable to the growth of a tumor such as esophageal cancer. While the idea is somewhat speculative, it will be interesting to see if other cases might be reported in the future that show metastases to IMLs or other benign tumors of carcinomas that do not typically affect bone.

In our search of the medical literature, metastasis to a pre-existing benign IML has not been reported before. The fact that our patient may be the first documented case perhaps reflects not just the rarity of the event but also the ease by which a subtle underlying lesion in bone may be overlooked and overshadowed by an aggressive tumor. In our case, we were fortunate in having images of the benign lesion in bone prior to its being affected by esophageal carcinoma, and we were thereby able to capture the process of metastasis to the lesion. It would be important for clinicians in the future to be sensitive to unusual characteristics of IMLs and other benign lesions of bone that might be a clue to the possibility of a concurrent secondary process within the lesion. Conversely, it may be equally noteworthy to observe radiographic signs of a pre-existing benign tumor that may have been all but obliterated by an obvious large bone metastasis. It is possible that studying these interactions might provide us with new and fresh clues as to the process of metastasis from malignant tumors.

\section{REFERENCES}

[1] M. F. Blacksin, N. Ende and J. Benevenia, "Magnetic Resonance Imaging of Intraosseous Lipomas: A RadiologicPathologic Correlation," Skeletal Radiology, Vol. 24, No. 1, 1995, pp. 37-41. doi:10.1007/BF02425945

[2] R. S. D. Campbell, A. J. Grainger, D. C. Mangham, I. Beggs, J. Teh and A. M. Davies, "Intraosseous Lipoma: Report of 35 New Cases and a Review of the Literature," Skeletal Radiology, Vol. 32, No. 4, 2003, pp. 209-222. doi:10.1007/s00256-002-0616-7

[3] J. Cuomo, F. H. Warren, L. M. Sanders and D. J. Beech, "Solitary Scapula Mass: Atypical Presentation of Esophageal Adenocarcinoma," American Surgeon, Vol. 64, No. 5, 1998, pp. 466-470.

[4] J. W. Milgram, "Malignant Transformation in Bone Lipomas," Skeletal Radiology, Vol. 19, No. 5, 1990, pp. 347352. doi:10.1007/BF00193088

[5] M. D. Murphey, J. F. Carroll, D. J. Flemming, T. L. Pope, F. H. Gannon and M. J. Kransdorf, "From the Archives of the AFIP: Benign Musculoskeletal Lipomatous Lesions," Radiographics, Vol. 24, No. 5, 2004, pp. 1433-1466. doi:10.1148/rg.245045120

[6] H. Ozdemir, Z. Bozgeyik, E. Kocakoc and O. Kalender, "MRI Findings of Intraosseous Lipoma: Case Report," Magnetic Resonance Imaging, Vol. 22, No. 2, 2004, pp. 281-284. doi:10.1016/i.mri.2003.08.032

[7] T. Propeck, M. A. Bullard, J. Lin, K. Doi and W. Martel, "Radiologic-Pathologic Correlation of Intraosseous Lipomas," American Journal of Roentgenology, Vol. 175, No. 3, 2000, pp. 673-678.

[8] L. E. Quint, L. M. Hepburn, I. R. Francis, R. I. Whyte and M. B. Orringer, "Incidence and Distribution of Distant Metastases from Newly Diagnosed Esophageal Carcinoma," Cancer, Vol. 76, No. 7, 1995, pp. 1120-1125. doi:10.1002/1097-0142(19951001)76:7<1120::AID-CNC R2820760704>3.0.CO;2-W

[9] D. C. Dahlin and K. K. Unni, "General Aspects and Data on 11087 Cases," 5th Edition, Lipponcott-Raven, Philadelphia, 1996.

[10] M. Yildirim, A. Sayın, F. Oztop, B. Doganavsargil and E. Goker, "Collision Tumor of the Scapula: A Case Report," The Internet Journal of Surgery, Vol. 17, No. 2, 2008, p. 20.

[11] J. T. Goodner and A. D. Turnbull, "Bone Metastases in Cancer of the Esophagus," American Journal of Roentgenology, Vol. 111, No. 2, 1971, pp. 365-367.

[12] A. Kapukaya, M. Subasi, N. Dabak and E. Ozkul, "Osseous Lipoma: Eleven New Cases and Review of the Literature," Acta Orthopaedica Belgica, Vol. 72, No. 5, 2006, pp. 603-614.

[13] J. W. Milgram, "Intraosseous lipomas: A Clinicopathologic Study of 66 Cases," Clinical Orthopaedics \& Related Research, Vol. 231, 1988, pp. 277-302. 